\title{
A COMPUTATIONAL METHOD FOR SOLVING TWO-DIMENSIONAL LINEAR FREDHOLM INTEGRAL EQUATIONS OF THE SECOND KIND
}

\author{
A. TARI ${ }^{\otimes 1,2}$ and S. SHAHMORAD ${ }^{3}$
}

(Received 16 August, 2007; revised 23 April, 2008)

\begin{abstract}
In this paper an expansion method, based on Legendre or any orthogonal polynomials, is developed to find numerical solutions of two-dimensional linear Fredholm integral equations. We estimate the error of the method, and present some numerical examples to demonstrate the accuracy of the method.
\end{abstract}

2000 Mathematics subject classification: 65R20.

Keywords and phrases: two-dimensional linear Fredholm integral equations, orthogonal polynomials.

\section{Introduction}

In recent years, many numerical methods of high accuracy have been developed for solving integral equations (see, for example, [2, 3, 6, 7, 9]). Much work has also been done on integro-differential equations (see [1, 4]). A great deal of this work is concerned with one-dimensional cases; however, methods for treating two-dimensional integral and integro-differential equations also deserve further study, as these equations have many applications in physics, mechanics and other applied sciences.

In this paper we develop expansion methods, based on orthogonal polynomials, for the numerical solution of two-dimensional linear Fredholm integral equations.

1.1. Legendre polynomials Consider the well-known Legendre polynomials which are defined by the recursive formula [5]

$$
\begin{aligned}
& p_{0}(x)=1, \\
& p_{1}(x)=x,
\end{aligned}
$$

\footnotetext{
${ }^{1}$ Faculty of Mathematical Science, University of Tabriz, Tabriz, Iran; e-mail: tari@ tabrizu.ac.ir.

${ }^{2}$ Department of Mathematics, University of Shahed, Tehran, Iran; e-mail: tari@ shahed.ac.ir.

${ }^{3}$ Faculty of Mathematical Science, University of Tabriz, Tabriz, Iran; e-mail: shahmorad@tabrizu.ac.ir.

(C) Australian Mathematical Society 2008, Serial-fee code 0334-2700/08
} 


$$
p_{n+1}(x)=\frac{2 n+1}{n+1} x p_{n}(x)-\frac{n}{n+1} p_{n-1}(x), \quad n=1,2, \ldots
$$

These polynomials are orthogonal on the interval $[-1,1]$ with respect to the weight function $\omega(x)=1$, but they are not orthonormal.

To normalize $\left\{p_{n}\right\}$, we set (see [3])

$$
p_{n}^{*}(x)=\sqrt{\frac{2 n+1}{2}} p_{n}(x), \quad n=0,1,2, \ldots
$$

Then, to shift $\left\{p_{n}^{*}\right\}$ to an arbitrary interval $[a, b]$, we set

$$
u_{n}(x)=\sqrt{\alpha} p_{n}^{*}(\alpha x-\beta), \quad n=0,1,2, \ldots,
$$

where $\alpha=2 /(b-a)$ and $\beta=(b+a) /(b-a)$.

Therefore $\left\{u_{n}\right\}$ is a set of orthonormal polynomials on the interval $[a, b]$ with respect to the weight function $\omega(x)=1$.

1.2. Chebyshev polynomials The Chebyshev polynomials are defined by

$$
\begin{gathered}
T_{0}(x)=1, \\
T_{1}(x)=x, \\
T_{n+1}(x)=2 x T_{n}(x)-T_{n-1}(x), \quad n=1,2, \ldots
\end{gathered}
$$

These polynomials are orthogonal on the interval $[-1,1]$ with respect to the weight function $\omega(x)=1 / \sqrt{1-x^{2}}$.

Normalization of these polynomials may be done by setting

$$
T_{0}^{*}(x)=\frac{1}{\sqrt{\pi}} T_{0}(x), \quad T_{n}^{*}(x)=\sqrt{\frac{2}{\pi}} T_{n}(x), \quad n=1,2, \ldots
$$

To shift $\left\{T_{n}^{*}\right\}$ to the arbitrary interval $[a, b]$, set

$$
u_{n}(x)=\sqrt{\alpha} T_{n}^{*}(\alpha x-\beta), \quad n=0,1,2, \ldots,
$$

where $\alpha=2 /(b-a)$ and $\beta=(b+a) /(b-a)$.

Thus $\left\{u_{n}\right\}$ is a set of orthonormal polynomials on the interval $[a, b]$ with respect to the weight function $\omega(x)=1 / \sqrt{1-(\alpha x-\beta)^{2}}$.

\section{Description of the method}

In this section, we describe a method for solving bivariate linear Fredholm integral equations by using Legendre polynomials. For Chebyshev polynomials the method is similar. 
Consider the integral equation

$$
\phi(x, t)-\int_{c}^{d} \int_{a}^{b} K(x, t, y, z) \phi(y, z) d y d z=f(x, t), \quad x \in[a, b], t \in[c, d]
$$

for which an approximate form is

$$
\phi_{N}(x, t)-\int_{c}^{d} \int_{a}^{b} K_{N}(x, t, y, z) \phi_{N}(y, z) d y d z=f_{N}(x, t),
$$

for $x \in[a, b]$ and $t \in[c, d]$, where $\phi_{N}, K_{N}$ and $f_{N}$ are approximations of $\phi, K$ and $f$, respectively.

Assume that $\left\{u_{n}\right\}_{n=0}^{N}$ and $\left\{v_{n}\right\}_{n=0}^{N}$ are the sets of Legendre polynomials on the intervals $[a, b]$ and $[c, d]$, respectively.

One can then write

$$
\begin{gathered}
\phi_{N}(x, t)=\sum_{i=0}^{N} \sum_{j=0}^{N} C_{i j} u_{i}(x) v_{j}(t), \quad x \in[a, b], t \in[c, d], \\
f_{N}(x, t)=\sum_{i=0}^{N} \sum_{i=0}^{N} f_{i j} u_{i}(x) v_{j}(t), \quad x \in[a, b], t \in[c, d]
\end{gathered}
$$

and

$$
\begin{array}{r}
K_{N}(x, t, y, z)=\sum_{i=0}^{N} \sum_{j=0}^{N} \sum_{l=0}^{N} \sum_{m=0}^{N} k_{i j l m} u_{i}(x) v_{j}(t) u_{l}(y) v_{m}(z), \\
x, y \in[a, b], \quad t, z \in[c, d],
\end{array}
$$

where the coefficients $f_{i j}$ and $k_{i j l m}$ are determined by

$$
f_{i j}=\int_{c}^{d} \int_{a}^{b} f(x, t) u_{i}(x) v_{j}(t) d x d t, \quad i, j=0,1, \ldots, N
$$

and

$$
\begin{array}{r}
k_{i j l m}=\int_{c}^{d} \int_{a}^{b} \int_{c}^{d} \int_{a}^{b} K(x, t, y, z) u_{i}(x) v_{j}(t) u_{l}(y) v_{m}(z) d x d t d y d z \\
i, j, l, m=0,1, \ldots, N
\end{array}
$$

while $C_{i j}$ are unknown coefficients which must be determined. 
Substituting $\phi_{N}(x, t), f_{N}(x, t)$ and $K_{N}(x, t, y, z)$ from (2.3), (2.4), (2.5) into (2.2) gives

$$
\begin{aligned}
& \sum_{i=0}^{N} \sum_{j=0}^{N} C_{i j} u_{i}(x) v_{j}(t) \\
& \quad-\int_{c}^{d} \int_{a}^{b} \int_{c}^{d} \int_{a}^{b}\left\{\left(\sum_{i=0}^{N} \sum_{j=0}^{N} \sum_{l=0}^{N} \sum_{m=0}^{N} k_{i j l m} u_{i}(x) v_{j}(t) u_{l}(y) v_{m}(z)\right)\right. \\
& \left.\quad \times\left(\sum_{r=0}^{N} \sum_{s=0}^{N} C_{r s} u_{r}(y) v_{s}(z)\right)\right\} d y d z \\
& =\sum_{i=0}^{N} \sum_{i=0}^{N} f_{i j} u_{i}(x) v_{j}(t)
\end{aligned}
$$

and the second term in the left-hand side of the above relation can be simplified to

$$
\begin{aligned}
& \sum_{i=0}^{N} \sum_{j=0}^{N} \sum_{l=0}^{N} \sum_{m=0}^{N} \sum_{r=0}^{N} \sum_{s=0}^{N} k_{i j l m} C_{r s} u_{i}(x) v_{j}(t) \int_{c}^{d} v_{m}(z) v_{s}(z) \int_{a}^{b} u_{l}(y) u_{r}(y) d y d z \\
& \quad=\sum_{i=0}^{N} \sum_{j=0}^{N} \sum_{l=0}^{N} \sum_{m=0}^{N} \sum_{r=0}^{N} \sum_{s=0}^{N} k_{i j l m} C_{r s} u_{i}(x) v_{j}(t) \delta_{m s} \delta_{l r} \\
& \quad=\sum_{i=0}^{N} \sum_{j=0}^{N} \sum_{l=0}^{N} \sum_{m=0}^{N} k_{i j l m} C_{l m} u_{i}(x) v_{j}(t) .
\end{aligned}
$$

Therefore, by substituting (2.9) into (2.8), we obtain

$$
\sum_{i=0}^{N} \sum_{j=0}^{N}\left\{C_{i j}-\sum_{l=0}^{N} \sum_{m=0}^{N} k_{i j l m} C_{l m}-f_{i j}\right\} u_{i}(x) v_{j}(t)=0,
$$

and so

$$
C_{i j}-\sum_{l=0}^{N} \sum_{m=0}^{N} k_{i j l m} C_{l m}-f_{i j}=0, \quad i, j=0,1, \ldots, N
$$

since both $\left\{u_{n}\right\}_{n=0}^{N}$ and $\left\{v_{n}\right\}_{n=0}^{N}$ are sets of basis functions.

Equivalently,

$$
\left(1-k_{i j i j}\right) C_{i j}-\sum_{l=0, l \neq i}^{N} \sum_{m=0, m \neq j}^{N} k_{i j l m} C_{l m}=f_{i j}, \quad i, j=0,1, \ldots, N .
$$

By solving this system, we obtain the unknowns $C_{i j}$ for $i, j=0,1, \ldots, N$ and, consequently, the approximate solution $\phi_{N}(x, t)$ of (2.3). 


\section{Error estimation}

It is known [3] that an orthogonal basis has the advantage that it guarantees convergence of the method based on it; in this section we estimate the size of the error.

For this purpose, we define the error function as

$$
e(x, t)=\phi(x, t)-\phi_{N}(x, t),
$$

where $\phi(x, t)$ is the exact solution and $\phi_{N}(x, t)$ is the approximate solution of the integral equation (2.1).

By substituting $\phi_{N}(x, t)$ in the integral equation (2.1), we obtain

$$
\phi_{N}(x, t)-\int_{c}^{d} \int_{a}^{b} K(x, t, y, z) \phi_{N}(y, z) d y d z=f(x, t)+h_{N}(x, t),
$$

where $h_{N}(x, t)$ is a perturbation term which can be found by substituting the computed solution $\phi_{N}(x, t)$ into the formula

$$
h_{N}(x, t)=\phi_{N}(x, t)-\int_{c}^{d} \int_{a}^{b} K(x, t, y, z) \phi_{N}(y, z) d y d z-f(x, t) .
$$

We proceed to find an approximation $e_{N}(x, t)$ to the error function $e(x, t)$ in the same way as we did earlier for the solution of equation (2.2).

By subtracting (3.2) from (2.1) and using (3.1), the error function $e(x, t)$ satisfies the equation

$$
e(x, t)-\int_{c}^{d} \int_{a}^{b} K(x, t, y, z) e(y, z) d y d z=-h_{N}(x, t) .
$$

It should be noted that in order to construct the approximation $e_{N}(x, t)$ to $e(x, t)$, only the right-hand side of equation (2.1) needs to be recomputed; thus, by solving the integral equation (3.3), we obtain an estimate for the error function (3.1).

\section{Numerical examples}

The following examples are given to demonstrate the accuracy of the method that we have presented.

EXAMPLE 1 .

$$
\begin{array}{r}
\phi(x, t)-\int_{-1}^{1} \int_{-1}^{1}\left(x \sin t+y e^{z}\right) \phi(y, z) d y d z=x e^{-t}+4 x \sin t-\frac{7}{3} \\
x, t \in[-1,1] .
\end{array}
$$

The exact solution is $\phi(x, t)=x e^{-t}-1$.

Table 1 shows the absolute error between the approximate solution and the exact solution at the points $(x, t)=(0.25 i, 0.25 i)$, for $i=-4,-3,-2,-1,0,1,2,3,4$, with $N=10$. 
TABLE 1. Errors $e(x, t)$ and $e_{N}(x, t)$ for $N=10$ at the points $(x, t)=(0.25 i, 0.25 i) i=-4,-3,-2$, $-1,0,1,2,3,4$.

\begin{tabular}{lll}
\hline$(x, t)$ & $e(x, t)$ & $e_{N}(x, t)$ \\
\hline$(-1,-1)$ & $0.7756 e-10$ & $0.7743 e-10$ \\
$(-0.75,-0.75)$ & $0.5092 e-11$ & $0.5116 e-11$ \\
$(-0.5,-0.5)$ & $0.2756 e-11$ & $0.2745 e-11$ \\
$(-0.25,-0.25)$ & $0.1209 e-11$ & $0.1208 e-11$ \\
$(0,0)$ & $0.1000 e-18$ & $0.5791 e-18$ \\
$(0.25,0.25)$ & $0.8407 e-12$ & $0.8386 e-12$ \\
$(0.5,0.5)$ & $0.2003 e-11$ & $0.1992 e-11$ \\
$(0.75,0.75)$ & $0.5596 e-11$ & $0.5618 e-11$ \\
$(1,1)$ & $0.7111 e-10$ & $0.7098 e-10$ \\
\hline
\end{tabular}

TABLE 2. Errors $e(x, t)$ and $e_{N}(x, t)$ for $N=10$ at $(x, t)=(0.5 i, 0.5 i) i=0,1,2,3,4,5,6$.

\begin{tabular}{lll}
\hline$(x, t)$ & $e(x, t)$ & $e_{N}(x, t)$ \\
\hline$(0,0)$ & $0.6112 e-13$ & $0.4939 e-13$ \\
$(0.5,0.5)$ & $0.8982 e-10$ & $0.8979 e-10$ \\
$(1,1)$ & $0.9181 e-11$ & $0.9527 e-11$ \\
$(1.5,1.5)$ & $0.1956 e-9$ & $0.1950 e-9$ \\
$(2,2)$ & $0.2982 e-9$ & $0.2976 e-9$ \\
$(2.5,2.5)$ & $0.2946 e-10$ & $0.2804 e-10$ \\
$(3,3)$ & $0.1233 e-9$ & $0.1218 e-9$ \\
\hline
\end{tabular}

TABLE 3. Errors $e(x, t)$ and $e_{N}(x, t)$ for $N=6$ at $(x, t)=(0.5 i, 0.5 j) i, j=0,1,2$.

\begin{tabular}{lll}
\hline$(x, t)$ & $e(x, t)$ & $e_{N}(x, t)$ \\
\hline$(0,0)$ & $0.133807 e-15$ & $0.134793 e-15$ \\
$(0,0.5)$ & $0.133814 e-15$ & $0.133971 e-15$ \\
$(0,1)$ & $0.133798 e-15$ & $0.130633 e-15$ \\
$(0.5,0)$ & $0.129039 e-7$ & $0.129168 e-7$ \\
$(0.5,0.5)$ & $0.229859 e-9$ & $0.229699 e-9$ \\
$(0.5,1)$ & $0.145827 e-7$ & $0.145969 e-7$ \\
$(1,0)$ & $0.258079 e-7$ & $0.258336 e-7$ \\
$(1,0.5)$ & $0.459718 e-9$ & $0.459398 e-9$ \\
$(1,1)$ & $0.291655 e-7$ & $0.291938 e-7$ \\
\hline
\end{tabular}

EXAMPLE 2 .

$$
\begin{array}{r}
\phi(x, t)-\int_{0}^{\pi} \int_{0}^{\pi}(x y+t z) \phi(y, z) d y d z=x \sin t-\left(\frac{2}{3} x+\frac{1}{2} t\right) \pi^{3} \\
x, t \in[0, \pi] .
\end{array}
$$

The exact solution is $\phi(x, t)=x \sin t$.

Table 2 shows the values of the absolute error at the points $(x, t)=(0.5 i, 0.5 i)$, for $i=0, \ldots, 6$, with $N=10$. 
TABLE 4. Errors $e(x, t)$ and $e_{N}(x, t)$ for $N=10$ at $(x, t)=(0.5 i, 0.5 j) i, j=0,1,2$.

\begin{tabular}{lll}
\hline$(x, t)$ & $e(x, t)$ & $e_{N}(x, t)$ \\
\hline$(0,0)$ & $0.4064 e-18$ & $0.8438 e-15$ \\
$(0,0.5)$ & $0.4556 e-19$ & $0.2463 e-15$ \\
$(0,1)$ & $0.6570 e-18$ & $0.1846 e-14$ \\
$(0.5,0)$ & $0.8130 e-14$ & $0.7950 e-14$ \\
$(0.5,0.5)$ & $0.7607 e-16$ & $0.1294 e-15$ \\
$(0.5,1)$ & $0.8804 e-14$ & $0.8409 e-14$ \\
$(1,0)$ & $0.1626 e-13$ & $0.1547 e-13$ \\
$(1,0.5)$ & $0.1522 e-15$ & $0.3802 e-15$ \\
$(1,1)$ & $0.1761 e-13$ & $0.1589 e-13$ \\
\hline
\end{tabular}

EXAMPLE 3.

$$
\begin{array}{r}
\phi(x, t)-\int_{0}^{1} \int_{0}^{1}(y \sin z+1) \phi(y, z) d y d z=x \cos t-\frac{1}{6} \sin (1)(\sin (1)+3) \\
x, t \in[0,1] .
\end{array}
$$

The exact solution is $\phi(x, t)=x \cos t$.

Tables 3 and 4 show the values of the absolute error at the points $(x, t)=(0.5 i, 0.5 j)$, for $i, j=0,1,2$, with $N=6$ and $N=10$, respectively.

\section{References}

[1] M. H. Aliabdi and S. Shahmorad, "A matrix formulation of the Tau method for Fredholm and Volterra linear integro-differential equations", The Korean J. Comput. Appl. Math. 9(2) (2002) 497-507.

[2] K. E. Atkinson, The numerical solution of integral equations of the second kind (Cambridge University Press, Cambridge, 1997).

[3] L. M. Delves and J. L. Mohamed, Computational methods for integral equations (Cambridge University Press, Cambridge, 1985).

[4] S. M. Hosseini and S. Shahmorad, "Tau numerical solution of Fredholm integro-differential equations with arbitrary polynomial bases", Appl. Math. Modeling 27 (2003) 145-154.

[5] M. K. Jain, S. R. K. Iyengar and R. K. Jain, Numerical methods for scientific and engineering computation (Wiley Eastern Limited, New Delhi, 1987).

[6] A. J. Jerri, Introduction to integral equations with applications (John Wiley \& Sons, New York, 1999).

[7] R. P. Kanwal, Linear integral equations (Academic Press, London, 1971).

[8] D. Kincaid and W. Cheney, Numerical analysis: mathematics of scientific computing (Brooks Cole Publishing Company, CA, 1990).

[9] R. Kress, Linear integral equations (Springer, Berlin, 1999). 